\title{
Cancer During Pregnancy: The Oncologist Overview
}

\author{
Adriana Hepner ${ }^{\mathrm{a}, \mathrm{g}}$, Daniel Negrini ${ }^{\mathrm{a}}$, Eliane Azeka Hase ${ }^{\mathrm{b}}$, Pedro Exman $^{\mathrm{c}}$, Laura Testa ${ }^{\mathrm{a}}$, \\ Angela F Trinconi ${ }^{\mathrm{d}}$, Jose Roberto Filassi ${ }^{\mathrm{d}}$, Rossana Pulcineli Vieira Francisco ${ }^{\mathrm{b}}$, \\ Marcelo Zugaib ${ }^{\mathrm{b}}$, Tracey L. O’Connor ${ }^{\mathrm{e}}$, Michael Gary Martin ${ }^{\mathrm{f}}$
}

\begin{abstract}
Although a rare and challenging condition, cancer during pregnancy should promptly be identified and treated. Not only standards of care guidelines for the underlying disease are taken into account, but also fetal safety might be weighted for clinical decisions. Frequent lack of experience and knowledge about this condition could lead to late diagnosis, imprecise management, suboptimal treatment and fetal and maternal harm. Therefore, this review aims to summarize the current evidence regarding the epidemiology, clinical presentation, diagnostic workup, staging and treatment, including novel treatment modalities for patients diagnosed with cancer during pregnancy.
\end{abstract}

Keywords: Cancer; Pregnancy; Fetus; Chemotherapy; Radiotherapy; Surgery; Targeted therapy; Immunotherapy

\section{Introduction}

Cancer during pregnancy is a rare event, occurring approximately once per 1,000 pregnancies annually, corresponding to $0.07 \%$ to $0.1 \%$ of all malignant tumors $[1,2]$. The most common malignancies associated with pregnancy are, in order of decreasing frequency, melanoma and breast cancer, cervical cancer, lymphomas and leukemias [3]. These histological types of malignancies are also among the most frequent can-

Manuscript submitted December 17, 2018, accepted December 31, 2018

aMedical Oncology Service, Instituto do Cancer do Estado de Sao Paulo, Universidade de Sao Paulo, Sao Paulo, Brazil

bepartment of Obstetrics and Gynecology, University of Sao Paulo Medical School, Sao Paulo, Brazil

${ }^{\mathrm{c}}$ Medical Oncology, Dana-Farber Cancer Institute, Boston, Massachusetts, USA

${ }^{\mathrm{d}}$ Mastology Service, Instituto do Cancer do Estado de Sao Paulo, Universidade de Sao Paulo, Sao Paulo, Brazil

eDepartment of Medicine, Roswell Park Comprehensive Cancer Center, The Jacobs School of Medicine, University of Buffalo, Buffalo, NY, USA

fDivision of Hematology and Oncology, University of Tennessee Health Science Center/West Cancer Center, Memphis, TN, USA

gCorresponding Author: Adriana Hepner, Instituto do Cancer do Estado de Sao

Paulo, Universidade de Sao Paulo, Sao Paulo, Brazil.

Email: drihepner@hotmail.com

doi: https://doi.org/10.14740/wjon1177 cers sites in nonpregnant women at younger ages. Melanoma, hematopoietic malignancies and lung cancer are the only cancers that have been reported to metastasize to the placenta and fetus, while melanoma accounts for almost one third of all cases [4]. Still, most of the data might be underrepresented due to difficulties involving diagnosis and data report, especially in underdeveloped countries.

The pathophysiology of cancer associated to pregnancy is not fully understood. However, hormonal changes, immunological suppression and increased permeability and vascularization are implied. As frequency is expected to increase, due to trends in delayed childbearing [5], health practitioners should be aware of particularities of the diagnose and multidisciplinary management of those women. This article will provide an updated review of the diagnosis, staging and treatment options for cancer during pregnancy in detail.

\section{Diagnosis and Staging Exams}

\section{Clinical presentation}

Signs and symptoms commonly seem in cancer may overlap and be masked by physiological changes that occur during pregnancy. Therefore, caretakers might easily attribute the symptoms of an undiagnosed cancer to pregnancy itself and do not proceed with further investigation when needed. In addition, concerns about the exposure of the fetus to inherent risks of complementary examination, such as ionizing radiation, contrasts and surgical/anesthetic procedures, might make physicians less prone to immediately proceed with the investigation of those symptoms [6, 7]. To make things worse, even in cases where the symptoms are properly investigated, there is still a higher chance of misdiagnose or false-negative results since pregnancy interferes with the sensibility and specificity of the diagnostic methods. Hence, unfortunately, in many cases the diagnosis of cancer during pregnancy is delayed [7].

\section{Imaging}

Intrauterine ionizing radiation exposure is known to be linked to teratogenicity, growth restriction, intellectual disability and even death [8]. Animal studies and follow-ups of the offspring of individuals exposed to atomic bomb explo- 
Table 1. Fetal Radiation Doses of the Most Frequent Used Imaging Methods in Oncology (Adapted From ACOG Committee Opinion. Guidelines for Diagnostic Imaging During Pregnancy and Lactation)

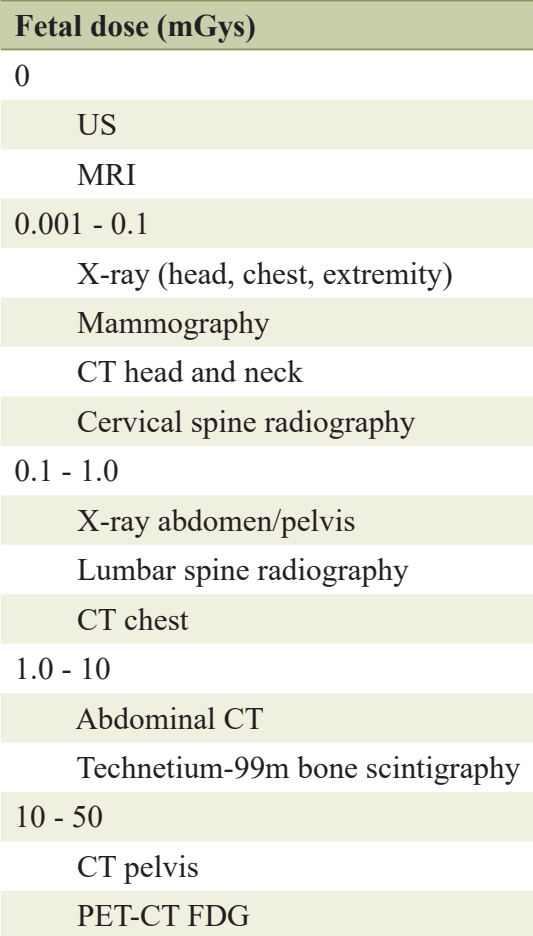

CT head and neck

Cervical spine radiography

$0.1-1.0$

X-ray abdomen/pelvis

Lumbar spine radiography

CT chest

$1.0-10$

Abdominal CT

Technetium-99m bone scintigraphy

$10-50$

CT pelvis

PET-CT FDG

Health care practitioners should consider avoiding complementary studies that deliver fetal doses higher than $0.1 \mathrm{mGys}$. Doses up from 10 mGys are considered prohibitive. US: ultrasound.

sions in Japan and the Chernobyl disaster corroborate that intrauterine ionizing radiation exposure increases lifetime cancer risk [8-10].

The gestational age plays an important role in determining the severity of the effect in consequence of radiation exposure. Exposures during the second to the eighth week of pregnancy (organogenesis stage) have a higher chance to induce major malformations [11,12]. Still, some fetal tissues continue to develop during all pregnancy, notably the central nervous system (especially during 8th - 15th week), and intrauterine exposure might result in cognitive impairment even in late pregnancy. Other major factor to be considered is the cumulative radiation dose received by the fetus. There is a clear correlation between higher doses of radiation and the severity of the impairment to the fetus, with an exponential risk in exposures that exceed 100 mGys, called the threshold dose $[8,13]$.

In order to avoid the effects of radiation to the fetus, nonradioactive imaging methods like magnetic resonance and ultrasound should be favored during pregnancy [6]. Many imaging methods deliver inferior ionizing radiation than the threshold dose of 100 mGys. Nevertheless, when adequate abdominal shielding is employed, they should not be withheld when necessary for proper oncologic management of the patient $[6,11,13,14]$. Computed tomography (CT) of the abdo- men and pelvis, fluoroscopic imaging used in procedures and some nuclear medicine techniques deliver higher doses to the fetus and should be avoided [11]. For example, radioactive iodine (iodine 131) has a long half-life of 8 days, crosses the placenta and can adversely affect the fetal thyroid. Whether for diagnostic or therapeutic treatment purposes, it should not be used during pregnancy [15]. Table 1 summarizes the fetal radiation doses of the most frequent imaging methods used in oncology.

Another important issue is the utilization of contrasts. Gadolineum is known to cross the placenta and has been proven teratogenic in animal studies, therefore it is not recommended, unless diagnostic performance is expected to improve fetal or maternal outcomes $[15,16]$. There is no consensus regarding the use of iodinated contrasts during pregnancy. Although iodinated contrast could cross the placenta and enter the fetal circulation, animal studies did not show teratogenic or mutagenic effects. Despite the lack of information in humans, it is generally not indicated during pregnancy [16].

For staging purposes, most oncology services recommend chest X-ray with abdominal shielding and abdominal ultrasound. In addition, magnetic resonance imaging (MRI) especially of the abdomen and bone structures, without contrast, can be ordered for evaluation of specific body parts whenever needed.

\section{Serum tumor markers}

Although serum tumor markers could be useful in the diagnosis, follow-up and management of cancer patients, they lack sensitivity and specificity during pregnancy, due to significant physiological variations in serum levels [17]. Commonly used tumor markers CA 15-3, SCC, CA 125 and AFP levels are increased in pregnancy and consequently are not reliable. On the other hand, CEA, CA 19-9, LDH, AMH, and HE-4 levels are not commonly increased in pregnancy and theoretically could be of additional help $[7,17,18]$. Some exceptions are inibin B, whose levels increase in the last trimester of a normal pregnancy, and LDH, which is a marker of hypertensive abnormalities related to pregnancy $[19,20]$.

\section{Surgical staging and diagnosis}

Surgical procedures and use of anesthetic drugs during pregnancy are considered safe $[19,20]$. The benefits of either open or video biopsies for diagnostic purposes often overcome the risks and should not be withheld [7].

Pregnancy itself may induce changes in tissues such as breast and uterine cervix that could mimic malignancy [7]. To reduce misdiagnosis, it is recommended to inform the pathologist about the pregnancy condition of the patient [21].

Radioactive nucleotides impregnated for detection of the sentinel lymph node cause low doses radiation exposure to the fetus, smaller than $5 \mathrm{mGys}$, and should be used if necessary, for adequate axilla management [14]. Blue dye is frequently associated with anaphylactic reactions and it is not routinely 
recommended during pregnancy [21].

\section{Surgical Treatment}

There is robust evidence regarding the safety of surgical procedures and use of anesthetics in pregnant women. The risk of maternal death is not increased, nor is it associated with birth defects [22-24]. Still, the risk of miscarriage is slightly elevated (1-2\%), especially during the first trimester. There is also an increase risk in the likelihood of low birth weight and premature delivery (1.5 - 2 times relative risk). The rate of complications and morbidity seems to be higher in major abdominal and pelvic procedures $[21,22,25]$. Anesthetic drugs have been used in pregnancy with a good safety record and there is no anesthetic drug listed as a proven teratogen $[21,26,27]$. Considering this minimal risk to the fetus and the potential benefits of the treatment, the recommendation is that oncological surgery can be carried out at any moment during pregnancy and should not be delayed if indicated [25]. On the other hand, most reconstructions, which are mainly aesthetic procedures, should be delayed until postpartum.

\section{Radiotherapy}

The typical dose in radiotherapy is usually in the range of 40 - 70 Gys, which is $10^{4}-10^{5}$ times higher than the doses utilized for diagnostic methods [12]. Considering such high values, there is a great concern about possible harms to the fetus and for this reason, radiotherapy is not routinely recommended during pregnancy and should be postponed until after childbirth whenever possible [22]. Still, in oncologic emergencies, where radiotherapy plays an important role (i.e. spinal cord compression, CNS metastases, superior vena cava syndrome) or in situations that waiting for pregnancy resolution would compromise the treatment efficacy, radiotherapy might be considered before the delivery $[22,28]$.

The resulting dose to the fetus depends on several factors including the total radiation dose, distance of the target lesion and the fetus, leakage from equipment, scattered radiation from collimator and beam modifiers and the radiation scattered within the patient [29]. The employment of shielding protects the fetus from the scattered dose in the room and increasing distance from the target to the fetus, reduces the radiation scattered within the patient that is received by the fetus. It is estimated that with adequate shielding, distances $>$ $30 \mathrm{~cm}$ from the edge of the field to the fetus, and with careful planning, the resulting dose could be kept in the range of 40 200 mGys. Therefore, some areas such as head, neck, extremities and breast could be irradiated with minimal radiation to the fetus and radiotherapy in those areas could be considered in selected cases [12]. Of note, the gestational age also plays an important role since the distance between the field's edge and the fetus might diminish according to the fundal highness, being an important variable when planning radiotherapy [29]. Abdominal and pelvic diseases cannot be irradiated without deleterious effects to the fetus and in cases where it cannot be delayed pregnancy termination should be considered [15].

\section{Systemic Treatment}

\author{
Cytotoxic chemotherapy
}

Most chemotherapeutic agents have low molecular weight and cross the placenta. Nearly all of them are known or suspected teratogens accordingly to animal studies. In humans the security data are limited due to the rarity of the cancerpregnancy condition and ethical issues related to conduction of clinical trials within this population [30-32]. Similar to ionizing radiation, the timing of exposure to antineoplastic drugs correlate with the severity of the injury to the fetus [30]. Chemotherapeutic insult to the embryo during the first 2 weeks of conception may interfere with the processes that facilitate implantation, leading to miscarriage and spontaneous abortion. However, if the embryo survives, it is expected to develop normally since its cells are undifferentiated and totipotent (all-or-nothing period). From the second to eighth week of pregnancy occurs the organogenesis, a critical phase with rapid differentiation and high vulnerability of the tissues to teratogenic insults. Exposures during this period are more likely to cause major malformations than in any other gestational period. Still, the central nervous system (CNS), genitals, eyes and the hematopoietic system continue to be vulnerable over the next few weeks of the first trimester. During the fetal phase that corresponds to the second and third trimester of pregnancy, the differentiated organs will complete their maturation and growth, and exposures in this period could interfere with this process and result in intrauterine growth restriction, low birth weight and preterm labor [31]. Retrospective data have shown a high incidence of major malformation in expositions during the first trimester, around $14 \%$, while during the second and third trimester the incidence was around 3\%, which is similar to that of the general population [32]. For this reason, chemotherapy should be avoided during the first trimester. If there is an urgent need to start treatment, pregnancy termination should be considered, and the mother should be properly advised of the high risk of teratogenicity in case she opts to carry on pregnancy [30-32]. Chemotherapy during the second and third trimester is considered relatively safe. Although there is no increase in malformations rates, obstetrical and neonatal complications may occur more frequently, and close pregnancy and fetal vitality monitoring should be offered [22, 30-32].

The physiological changes that occur during pregnancy such as hypervolemia, enhanced renal/hepatic elimination and reduced albumin levels may interfere with the pharmacokinetics of the chemotherapeutics. However, since there are no studies regarding the efficacy of treatment regimens in this context, there is no dose adjustment preconized and current recommendation is to utilize the same dose as used in non-pregnant women $[30,31]$. Special attention is required to recalculate dosing at each cycle as patient weight usually increases during pregnancy.

To reduce hematological risk to the fetus, it is recommended to stop chemotherapy 3 weeks before the expected date of delivery or after $\geq 35$ weeks of pregnancy. This recommendation is to avoid that the nadir of the treatment coincides 
Table 2. Studies of Long-Term Follow-Up of Individuals Exposed to Intrauterine Chemotherapy

\begin{tabular}{|c|c|c|c|}
\hline & $\mathbf{N}$ & Years of follow-up & Findings \\
\hline $\begin{array}{l}\text { Sokal et al } \\
\text { (1960) [34] }\end{array}$ & 17 & $2-9.5$ & All individuals reported to have no abnormalities. \\
\hline $\begin{array}{l}\text { Reynoso et al } \\
(1987)[35]\end{array}$ & 6 & $1-16$ & $\begin{array}{l}\text { One individual with two neoplasias (thyroid and neuroblastoma) } \\
\text { and low IQ. All other individuals have no abnormalities. }\end{array}$ \\
\hline $\begin{array}{l}\text { Nulman et al } \\
(2001)[36]\end{array}$ & 111 & $1 m-22$ & Neurocognitive evaluation normal in all individuals. \\
\hline $\begin{array}{l}\text { Amant et al } \\
(2012)[38]\end{array}$ & 70 & $1.4-17.5$ & $\begin{array}{l}\text { Individuals exposed to chemotherapy during the second and third trimester. } \\
\text { No difference in comparing to general population in regard to general health, } \\
\text { development, cognition, behavior, cardiologic or neurologic diseases. }\end{array}$ \\
\hline
\end{tabular}

with the birth date and to reduce maternal risk of infections and bleeding. Weekly regimens should be preferred since they are associated with lower hematological toxicity and shorter nadir periods [22].

It is also important to note that chemotherapeutic agents differ in their teratogenic potential and some specific drugs such as methotrexate, dacarbazine, cyclophosphamide and cytarabine are associated with higher teratogenic potential [30, 32, 33].

Data regarding long-term outcomes of individuals exposed to chemotherapy during intrauterine life are also limited, but the few studies available showed that those individuals have normal growth and development (Table 2) [32, 34-38].

\section{Endocrine treatment}

Considering the high frequency of hormonal expression (positivity of estrogen receptor and progesterone receptor) in pregnancy-associated breast cancer, inquiries about the utilization of endocrine therapy in this scenario are pertinent. The physiological changes necessary for a healthy pregnancy and fetal development are mainly hormone mediated. Consequently, the utilization of drugs that block estrogen and progesterone production or action might interfere with those physiological processes. Of note, tamoxifen, the most utilized drug in the pre-menopausal context, is teratogenic in animals and has been associated with birth defects in children of women who inadvertently have utilized the medication during pregnancy.
Hence, endocrine treatment is contraindicated [39, 40].

\section{Molecularly targeted agents}

Drugs that target specific molecules involved in the growth and spread of malignant cells are increasingly being used in modern oncology practice. Most of these drugs are considered new on the market and have no accumulated data of its effects while exposition during pregnancy. Therefore, we discussed the available evidence and concerns about some of the drugs of this class, which were used more widely and long enough to have available data during pregnancy (Table 3 ).

Imatinib, which was the first molecule to provide the proof of principle that targeting an aberrant tyrosine kinase responsible for the uncontrolled cell cycle progression could control tumor progression in chronic myeloid leukemia, has been associated with an increase in spontaneous abortion and major malformations, especially exencephaly, encephalopathies and abnormalities in the skull bones. Of note, the malformations observed in humans are similar to those described in animals, reinforcing the teratogenic potential of this medication $[32,41$, 42].

Rituximab, a monoclonal anti-CD20 antibody, has been used in pregnancy with a good safety record. The rate of malformations in inadvertently exposures during pregnancy was not higher than that observed in the general population. The most relevant concern about its usage while on pregnancy is its potential to cause immunosuppression by B-cell depletion

Table 3. Pregnancy Risk Classification of Some of the Most Used Targeted Therapies and Immunotherapies in Medical Oncology

\begin{tabular}{|c|c|c|}
\hline Drug & Pregnancy/neonatal complication & Teratogenic \\
\hline Imatinib & Not associated & Yes \\
\hline Rituximab & Neonatal B-cell depletion & Not associated \\
\hline Trastuzumab & Oligohidramnios & Not associated \\
\hline Lapatinib & Possibly oligohydramnios & Yes (animal studies) \\
\hline Bevacizumab & Possibly pre-eclampsia & No data \\
\hline Ipilimumab & Abortion, stillbirths, premature delivery and higher incidence of infant mortality & Not associated \\
\hline PD-1/PD-L1 inhibitors & Abortion, stillbirths, premature delivery and higher incidence of infant mortality & Not associated \\
\hline
\end{tabular}


in neonates, highlighting the necessity of a multidisciplinary care team, including an attentive neonatal physician [32, 43].

Trastuzumab, an anti-HER2 antibody, has been clearly associated with oligohydramnios in pregnant women exposed to this medication, being considered proscribed.

There is no evidence regarding the effects of bevacizumab in pregnancy. However, it is known to cause hypertension and proteinuria; and for that reason, it is hypothesized that it might induce pre-eclampsia as well [44].

\section{Immunotherapy}

Since the mother and fetus are not genetically identical, it is necessary for the mother to develop an immune tolerance towards the fetus for a successful pregnancy [45]. Many immunomodulatory pathways are involved to create this tolerance. The immune checkpoints seem to have a key role in this process, more importantly via programmed cell death ligand 1 (PD-1/PDL-1), although CTLA-4 is also involved. Their inhibition could theoretically result in an immune response against the fetus [46]. In addition, available drugs that inhibit these immune checkpoints are IgG4 antibodies, which can cross the placenta and may cause direct toxicity to the fetus [47]. In animal models, anti-PD-1/PD-L1 and anti-CTLA-4 inhibitors during pregnancy were associated with an increase in abortion rates, stillbirths, premature delivery and higher incidence of infant mortality, especially when utilized during the third trimester. These studies have not shown an increase in the rate of malformations. Still, it is important to emphasize that these studies utilized doses that were much higher than those utilized therapeutically, and no human trials have been conducted so far [47-49]. The incorporation of these drugs into clinical practice is recent and there is no sufficient data to speculate about their security in humans. For the time being, the utilization of these drugs during pregnancy is not recommended.

\section{Support medications}

Some non-antineoplastic agents routinely used in oncology practice may also be a cause of concern during pregnancy.

Bisphosphonates inhibit osteoclastic activity, interfering with the calcium homeostasis, diminishing its levels in maternal blood. Utilization of this class of medication during pregnancy may reduce the calcium delivered to the fetus and induce skeletal malformations (reduced bone growth), low birth weight as demonstrated in animal models [50-52]. Also, bisphosphonates could induce maternal hypocalcemia and adversely affect parturition by diminishing uterine contraction [53]. For these reasons, utilization of bisphosphonates is generally contraindicated in pregnancy. New compounds, such as cimadronate, are being developed and have shown a good safety profile in animal studies [52].

The security of granulocyte colony stimulation factors (GCS-F) in pregnancy is also not fully established. There is evidence that it can cross the placenta and animal studies ob- served an increased rate of spontaneous abortion and low birth weight with no increase in malformations. Despite this, these drugs have been utilized during human pregnancies with good safety record [54]. Considering the risk and potential benefits, the recommendation is to administer GCS-F only in cases of severe neutropenia.

\section{Conclusions}

The identification of cancer during pregnancy is challenging. Physiological changes that occur during pregnancy can delay proper investigation of an underlying neoplasm. Consequently, a multi-disciplinary team work is needed to promptly assess gestational age, evaluate fetus viability and growth, while defining the best maternal diagnostic strategy. Always consider referral to centers with high risk obstetrical unit. Non-ionizing imaging methods are preferred, including MRI and ultrasound, aiming to reduce fetal radiation exposure. Surgery can be safely carried out at any moment during pregnancy and should not be delayed if indicated. Typically, radiotherapy is not indicated in this scenario, with exception of oncological emergencies, where maternal risk is imposed. Other indications should be discussed individually, by assessing and considering the resulting dose to the fetus. Systemic treatments should, ideally, follow as closely as possible, standard protocols for nonpregnant patients and, at the same time, securing fetal safety. Unfortunately, not many medications can be safely used during pregnancy and mother should be exhaustive thought about potential risks and complications of those systemic treatments. Therapeutic abortion can be an option in a few cases, when legislation permits.

To conclude, the treatment landscape in oncology is rapidly evolving. Nevertheless, few modern therapies are being incorporated for pregnant patients. Pharmacokinetics, pharmacodynamics and breast milk concentration studies of those new drugs, including small molecules and immunotherapy are needed. Additionally, medical community ought to constantly gather data on the long-term outcome of those children exposed to treatments in utero.

\section{Conflict of Interest}

The authors have no conflicts of interest to disclose related to this paper.

\section{Funding}

No funding was received for the preparation of the current manuscript.

\section{References}

1. Donegan WL. Cancer and pregnancy. CA Cancer J Clin. $1983 ; 33(4): 194-214$. 
2. Jhaveri MB, Driscoll MS, Grant-Kels JM. Melanoma in pregnancy. Clin Obstet Gynecol. 2011;54(4):537-545.

3. Pavlidis NA. Coexistence of pregnancy and malignancy. Oncologist. 2002;7(4):279-287.

4. Alexander A, Samlowski WE, Grossman D, Bruggers CS, Harris RM, Zone JJ, Noyes RD, et al. Metastatic melanoma in pregnancy: risk of transplacental metastases in the infant. J Clin Oncol. 2003;21(11):2179-2186.

5. Theriault RL, Gwyn KMEH, Hortobagyi GN. Cancer Treatment During Pregnancy. In: Kufe DW, Pollock RE, Weichselbaum RR, et al., editors. Holland-Frei Cancer Medicine. 6th edition. Hamilton (ON): BC Decker; 2003.

6. de Haan J, Vandecaveye V, Han SN, Van de Vijver KK, Amant F. Difficulties with diagnosis of malignancies in pregnancy. Best Pract Res Clin Obstet Gynaecol. 2016;33:19-32.

7. Andersson TM, Johansson AL, Fredriksson I, Lambe M. Cancer during pregnancy and the postpartum period: A population-based study. Cancer. 2015;121(12):2072-2077.

8. Streffer C, Shore R, Konermann G, Meadows A, Uma Devi P, Preston Withers J, Holm LE. ICRP publication 90 - Biological Effects after Prenatal Irradiation (Embryo and Fetus). Ann ICRP (International Commission on Radiological Protection). 2003;33:205-206.

9. Schull WJ, Otake M. Cognitive function and prenatal exposure to ionizing radiation. Teratology. 1999;59(4):222226.

10. Petridou E, Trichopoulos D, Dessypris N, Flytzani V, Haidas S, Kalmanti M, Koliouskas D, et al. Infant leukaemia after in utero exposure to radiation from Chernobyl. Nature. 1996;382(6589):352-353.

11. Fenig E, Mishaeli M, Kalish Y, Lishner M. Pregnancy and radiation. Cancer Treat Rev. 2001;27(1):1-7.

12. Gilman EA, Kneale GW, Knox EG, Steward AM. Pregnancy, X-rays and childhood cancers: effect of exposure, age and radiation dose. J Radiol Prot. 1988;8:3-8.

13. Dauer LT, Thornton RH, Miller DL, Damilakis J, Dixon RG, Marx MV, Schueler BA, et al. Radiation management for interventions using fluoroscopic or computed tomographic guidance during pregnancy: a joint guideline of the Society of Interventional Radiology and the Cardiovascular and Interventional Radiological Society of Europe with Endorsement by the Canadian Interventional Radiology Association. J Vasc Interv Radiol. 2012;23(1):19-32.

14. Kal HB, Struikmans H. Radiotherapy during pregnancy: fact and fiction. Lancet Oncol. 2005;6(5):328-333.

15. Committee on Obstetric P. Committee Opinion No. 723: Guidelines for diagnostic imaging during pregnancy and lactation. Obstet Gynecol. 2017;130(4):e210-e216.

16. Cohan RH, Davenport M, Dillman JR, Ellis JH, Hartman RP, Herts BR, Jafri SZ, et al. The American College of Radiology. Manual on Contrast Media. Version 7.0. 2010. p. 81 .

17. Han SN, Lotgerink A, Gziri MM, Van Calsteren K, Hanssens M, Amant F. Physiologic variations of serum tumor markers in gynecological malignancies during pregnancy: a systematic review. BMC Med. 2012;10:86.

18. Sarandakou A, Protonotariou E, Rizos D. Tumor markers in biological fluids associated with pregnancy. Crit Rev Clin Lab Sci. 2007;44(2):151-178.

19. Petraglia F, Luisi S, Benedetto C, Zonca M, Florio P, Casarosa E, Volpe A, et al. Changes of dimeric inhibin B levels in maternal serum throughout healthy gestation and in women with gestational diseases. J Clin Endocrinol Metab. 1997;82(9):2991-2995.

20. Talwar P, Kondareddy T, Shree P. LDH as prognostic marker in hypertensive pregnancy. International Journal of Reproduction, Contracept, Obstetrics and Gynecology. 2017;6(6):2444-2446.

21. Amant F, Deckers S, Van Calsteren K, Loibl S, Halaska $\mathrm{M}$, Brepoels L, Beijnen J, et al. Breast cancer in pregnancy: recommendations of an international consensus meeting. Eur J Cancer. 2010;46(18):3158-3168.

22. Peccatori FA, Azim HA, Jr., Orecchia R, Hoekstra HJ, Pavlidis N, Kesic V, Pentheroudakis G, et al. Cancer, pregnancy and fertility: ESMO Clinical Practice Guidelines for diagnosis, treatment and follow-up. Ann Oncol. 2013;24(Suppl 6):vi160-170.

23. Duncan PG, Pope WD, Cohen MM, Greer N. Fetal risk of anesthesia and surgery during pregnancy. Anesthesiology. 1986;64(6):790-794.

24. Cohen-Kerem R, Railton C, Oren D, Lishner M, Koren G. Pregnancy outcome following non-obstetric surgical intervention. Am J Surg. 2005;190(3):467-473.

25. Pentheroudakis G, Pavlidis N. Cancer and pregnancy: poena magna, not anymore. Eur J Cancer. 2006;42(2):126140.

26. Upadya M, Saneesh PJ. Anaesthesia for non-obstetric surgery during pregnancy. Indian J Anaesth. 2016;60(4):234241.

27. Ni Mhuireachtaigh R, O'Gorman DA. Anesthesia in pregnant patients for nonobstetric surgery. J Clin Anesth. 2006;18(1):60-66.

28. Chen Z, King W, Pearcey R, Kerba M, Mackillop WJ. The relationship between waiting time for radiotherapy and clinical outcomes: a systematic review of the literature. Radiother Oncol. 2008;87(1):3-16.

29. Stovall M, Blackwell CR, Cundiff J, Novack DH, Palta JR, Wagner LK, Webster EW, et al. Fetal dose from radiotherapy with photon beams: report of AAPM Radiation Therapy Committee Task Group No. 36. Med Phys. 1995;22(1):63-82.

30. Cardonick E, Iacobucci A. Use of chemotherapy during human pregnancy. Lancet Oncol. 2004;5(5):283-291.

31. Koren G, Carey N, Gagnon R, Maxwell C, Nulman I, Senikas V. Cancer chemotherapy and pregnancy. J Obstet Gynaecol Can. 2013;35(3):263-278.

32. National Toxicology P. NTP Monograph: Developmental Effects and Pregnancy Outcomes Associated With Cancer Chemotherapy Use During Pregnancy. NTP Monogr. 20132):i-214.

33. Azim HA, Jr., Peccatori FA, Pavlidis N. Treatment of the pregnant mother with cancer: a systematic review on the use of cytotoxic, endocrine, targeted agents and immunotherapy during pregnancy. Part I: Solid tumors. Cancer Treat Rev. 2010;36(2):101-109.

34. Sokal JE, Lessmann EM. Effects of cancer chemother- 
apeutic agents on the human fetus. J Am Med Assoc. 1960;172:1765-1771.

35. Reynoso EE, Shepherd FA, Messner HA, Farquharson HA, Garvey MB, Baker MA. Acute leukemia during pregnancy: the Toronto Leukemia Study Group experience with long-term follow-up of children exposed in utero to chemotherapeutic agents. J Clin Oncol. 1987;5(7):10981106.

36. Nulman I, Laslo D, Fried S, Uleryk E, Lishner M, Koren G. Neurodevelopment of children exposed in utero to treatment of maternal malignancy. Br J Cancer. 2001;85(11):1611-1618.

37. Aviles A, Neri N. Hematological malignancies and pregnancy: a final report of 84 children who received chemotherapy in utero. Clin Lymphoma. 2001;2(3):173-177.

38. Amant F, Van Calsteren K, Halaska MJ, Gziri MM, Hui W, Lagae L, Willemsen MA, et al. Long-term cognitive and cardiac outcomes after prenatal exposure to chemotherapy in children aged 18 months or older: an observational study. Lancet Oncol. 2012;13(3):256-264.

39. Barthelmes L, Gateley CA. Tamoxifen and pregnancy. Breast. 2004;13(6):446-451.

40. Braems G, Denys H, De Wever O, Cocquyt V, Van den Broecke R. Use of tamoxifen before and during pregnancy. Oncologist. 2011;16(11):1547-1551.

41. Pye SM, Cortes J, Ault P, Hatfield A, Kantarjian H, Pilot $\mathrm{R}$, Rosti G, et al. The effects of imatinib on pregnancy outcome. Blood. 2008;111(12):5505-5508.

42. Abruzzese E, Trawinska MM, Perrotti AP, De Fabritiis P. Tyrosine kinase inhibitors and pregnancy. Mediterr J Hematol Infect Dis. 2014;6(1):e2014028.

43. Chakravarty EF, Murray ER, Kelman A, Farmer P. Pregnancy outcomes after maternal exposure to rituximab. Blood. 2011;117(5):1499-1506.

44. Robinson AA, Watson WJ, Leslie KK. Targeted treatment using monoclonal antibodies and tyrosine-kinase inhibitors in pregnancy. Lancet Oncol. 2007;8(8):738-743.
45. Luppi P. How immune mechanisms are affected by pregnancy. Vaccine. 2003;21(24):3352-3357.

46. Ghorani E, Kaur B, Fisher RA, Short D, Joneborg U, Carlson JW, Akarca A, et al. Pembrolizumab is effective for drug-resistant gestational trophoblastic neoplasia. Lancet. 2017;390(10110):2343-2345.

47. Poulet FM, Wolf JJ, Herzyk DJ, DeGeorge JJ. An Evaluation of the Impact of PD-1 Pathway Blockade on Reproductive Safety of Therapeutic PD-1 Inhibitors. Birth Defects Res B Dev Reprod Toxicol. 2016;107(2):108-119.

48. Johnson DB, Sullivan RJ, Menzies AM. Immune checkpoint inhibitors in challenging populations. Cancer. 2017;123(11):1904-1911.

49. D'Addio F, Riella LV, Mfarrej BG, Chabtini L, Adams LT, Yeung M, Yagita H, et al. The link between the PDL1 costimulatory pathway and Th17 in fetomaternal tolerance. J Immunol. 2011;187(9):4530-4541.

50. Stathopoulos IP, Liakou CG, Katsalira A, Trovas G, Lyritis GG, Papaioannou NA, Tournis S. The use of bisphosphonates in women prior to or during pregnancy and lactation. Hormones (Athens). 2011;10(4):280-291.

51. Patlas N, Golomb G, Yaffe P, Pinto T, Breuer E, Ornoy A. Transplacental effects of bisphosphonates on fetal skeletal ossification and mineralization in rats. Teratology. 1999;60(2):68-73.

52. Okazaki A, Matsuzawa T, Takeda M, York RG, Barrow PC, King VC, Bailey GP. Intravenous reproductive and developmental toxicity studies of cimadronate (YM175), a novel bisphosphonate, in rats and rabbits. J Toxicol Sci. 1995;20(Suppl 1):1-13.

53. Minsker DH, Manson JM, Peter CP. Effects of the bisphosphonate, alendronate, on parturition in the rat. Toxicol Appl Pharmacol. 1993;121(2):217-223.

54. Cardonick E, Irfan F, Torres N. The use of Neupogen (Filgrastim) or Neulasta (Pegfilgrastim) during pregnancy when chemotherapy is indicated for maternal cancer treatment. Journal of Cancer Therapy. 2012;3:157-161. 